\title{
Radioactive gold nanoparticles show promise for tumour shrinkage
}

Researchers at the University of Missouri have developed radioactive gold nanoparticles that are specifically targeted to prostate cancer cells by their conjugation with epigallocatechin-gallate (EGCg). They report the ability of these nanoparticles to reduce tumour volume in Proceedings of the National Academy of Sciences of the United States of America.

Radiation therapy for prostate cancer is varied but rife with limitations; current modalities suffer from imprecise dose delivery (intensity-modulated radiation therapy), low efficacy (stereotactic body radiotherapy and external beam radiation therapy), or staggering costs (proton therapy). Brachytherapy treatment with radioactive seeds has been somewhat successful, but the size of the seeds $(50-100 \mu \mathrm{m})$ is thought to hinder access to tumour vasculature (150-300 nm). Radioactive nanoparticles can penetrate smaller spaces and thus have the potential to maximize therapeutic payload.

Radioactive gold $\left({ }^{198} \mathrm{Au}\right)$ is inherently therapeutic and has radiochemical properties that make it an attractive option for prostate cancer-a beta energy emission of $0.96 \mathrm{MeV}$ combined with a penetration range of $11 \mathrm{~mm}$ in tissue allows the destruction of prostate cancer cells while minimizing radiation exposure to the surrounding tissues. Investigators hypothesised that conjugation of ${ }^{198} \mathrm{Au}$ with EGCg, an antioxidant found in green tea, would provide two further advantages. First, that EGCg might be effective at reducing radioactive gold salt to radioactive gold nanoparticles without the need for toxic chemicals, and second, that the high affinity of EGCg for the laminin receptor Lam 67R would provide additional targeting to prostate cancer cells, which are known to express high levels of Lam 67R.

Researchers were indeed able to produce EGCg-functionalized radioactive gold nanoparticles ( ${ }^{198} \mathrm{AuNP}-\mathrm{EGCg}$ ) with $>99 \%$ yields owing to the strong reduction capabilities of EGCg. They then demonstrated the specificity of the nanoparticles for Lam 67R in receptor

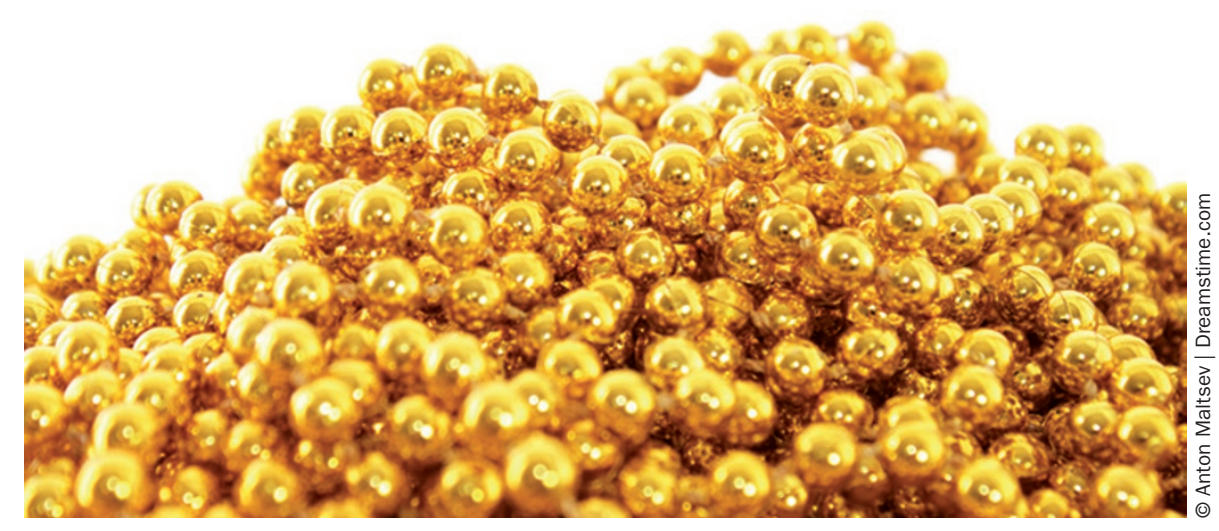

saturation experiments. Blocking Lam $67 \mathrm{R}$ on PC-3 cells by pretreatment with either laminin (the native ligand) or a specific antibody against Lam 67R (MLuC5) resulted in receptor saturation and a concomitant reduction in the internalization of nonradioactive surrogate nanoparticles.

To assess the therapeutic efficacy of ${ }^{198} \mathrm{AuNP}-\mathrm{EGCg}$, the research team transferred their attention to a mouse model of prostate cancer-severe combined immunodeficient mice with unilateral prostate tumours derived from human PC-3 cells. $24 \mathrm{~h}$ after intratumoural injection of ${ }^{198} \mathrm{AuNP}-\mathrm{EGCg}, 70 \%$ of radioactivity was retained within prostate tumours with minimal leakage to other organs and the blood. Within 1 week of administration, tumour growth had slowed in treated animals compared to controls (injected with Dulbecco's phosphate-buffered saline), and after 28 days tumour volume was $80 \%$ lower in the radiotherapy group than in control animals $(P=0.01)$.

The authors hope their 'trojan horse' approach to the delivery of radioactive gold to the prostate might one day be a useful tool for the treatment of human prostate cancer, and plan to extend their studies accordingly.

Sarah Payton

Original article Shukla, R. et al. Laminin receptor specific therapeutic gold nanoparticles ( ${ }^{198} \mathrm{AuNP}$-EGCg) show efficacy in treating prostate cancer. Proc. Natl Acad. Sci. USA doi:10.1073/pnas.1121174109 